\title{
Dentist creates free smartphone resource for DCTs and junior staff
}

Julian Woolley, who is about to become an orthodontic trainee, has built and released a free smartphone application aimed at foundation dentists, dental core trainees (DCTs) and other junior hospital staff. The app, SHO:me, aims to provide advice and a resource for guidelines and best practice in primary, secondary and tertiary care.

Explaining why he created the app, Julian said: 'Having worked in primary and secondary care, I often found it frustrating that guidelines and other important resources were located in different places eg handbooks, websites etc. No one wants to carry a handbook around when they're doing a 12-hour shift, and if they do, they'll likely leave it on a ward and lose it. I wanted an easily accessible digital handbook where I could find information straight away. I also wanted to pass this information on to the next cohort of foundation dentists and DCTs so that they don't feel like a "deer in headlights" when they start their new posts'.

The app is split into multiple easy-to-navigate sections and provides the user with a digestible resource in an accessible format. It is kept up to date with the latest guidance and new information is continually added by a team of contributors. Julian hopes to build more features into the app in the future, enabling young dentists to make better evidencebased decisions when treatment planning and feel more comfortable in the hospital environment.

Julian was inspired by apps designed for other medical specialties, that help junior doctors when they move into a new rotation. In order to put the app together, he taught himself how to code during the second lockdown, when the gym and rowing club were shut.

Julian said: 'I was fortunate that I was able to bring together a good team of editors and contributors to help collate and produce material. I also secured some funding from the British Orthodontic Society which enabled me to release the app for free and cover the start-up costs. It's really rewarding when I see users from all over the globe accessing the app and we have received lots of positive feedback so far'.

To download the app, simply search 'SHO me' on the Apple App Store or Google Play Store. The app has been designed to provide qualified dentists and dental professionals with evidence-based advice and guidance and is not intended to be used by the general public.

\section{Moisturising protection for your hands}

Many handwashes and hand sanitisers can dry out skin, removing its natural oils and creating dry, cracked areas that could be prone to infection.

Help to protect the hands of your patients and staff with the Steri-7 Xtra hand preparation solution range from Initial Medical, which includes hand rub and wash. These products offer exceptional protection and kill $99.99 \%$ of pathogens including coronavirus [tested against feline coronavirus, a surrogate virus for coronavirus] and stay active for up to three hours after application [source: Steri-7]. They offer a high level of hygienic protection to anything from pre-operative surgical scrubs to general use in kitchens, washrooms and toilets.

Hand safe and dermatologically tested, the solutions are also moisturising and nourish the hands as they protect, making them the perfect option for repeated use.

Find out more by contacting the team today.

For further information, visit www.initial.co.uk/medical or Tel: 08708504045 .

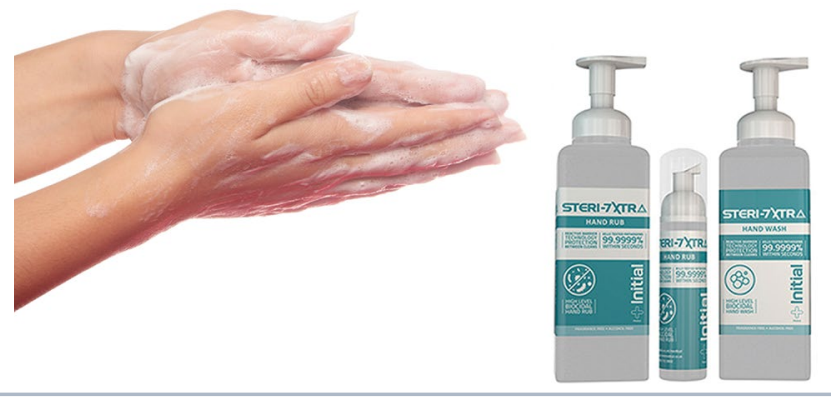

\section{The only $100 \%$ customised option}

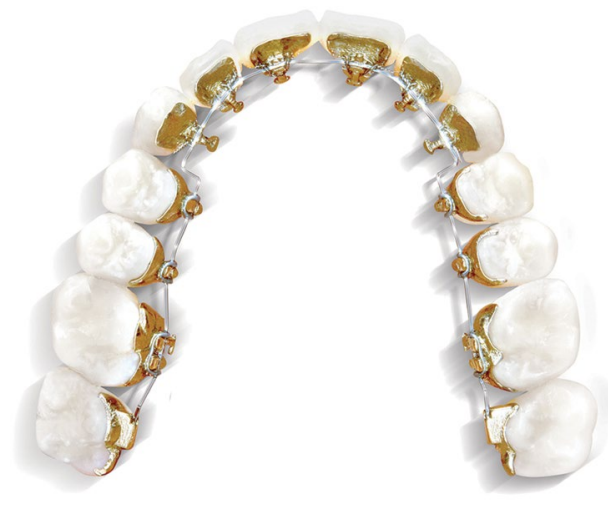

When offering your patients orthodontic treatment, you want to be confident that you can provide a solution that is fully tailored to their needs.

With the Incognito Appliance System from 3M Oral Care, you can guarantee bespoke, predictable treatment every time.

The only $100 \%$ customised, invisible fixed orthodontic appliance system on the market, the Incognito Appliance System features customised brackets, archwires and bonding trays, helping to deliver a highly personalised approach. Plus, as the system is placed lingually, it ensures patients can continue to live their daily lives with confidence.

To find out more, contact $3 \mathrm{M}$.

For more information, call 08458734066 or visit www.3m.co.uk/ incognito. $3 \mathrm{M}$ representatives remain contactable by phone or via video conferencing

$3 \mathrm{M}$ and Incognito are trademarks of the $3 \mathrm{M}$ Company. 